\title{
Multivariate Analysis of Attenuated Total Reflection Fourier Transform Infrared (ATR FT-IR) Spectroscopic Data to Confirm Phase Partitioning in Methacrylate-Based Dentin Adhesive
}

\author{
Qiang Ye ${ }^{a,{ }^{*}}$, Ranganathan Parthasarathy ${ }^{\mathrm{a}}$, Farhana Abedin ${ }^{\mathrm{a}}$, Jennifer S. Laurence ${ }^{\mathrm{b}}$, Anil \\ Misra $^{a, c}$, and Paulette Spencer ${ }^{a, d}$ \\ aUniversity of Kansas, Bioengineering Research Center, 1530 W. 15th Street, Lawrence, KS \\ 66045-7609, USA \\ bUniversity of Kansas, Department of Pharmaceutical Chemistry, 2095 Constant Avenue, \\ Lawrence, KS 66047-3729, USA \\ 'University of Kansas, Department of Civil Engineering, 1530 W. 15th Street, Lawrence, KS \\ 66045-7609, USA \\ dUniversity of Kansas, Department of Mechanical Engineering, 1530 W. 15th Street, Lawrence, \\ KS 66045-7609, USA
}

\section{Abstract}

Water is ubiquitous in the mouths of healthy individuals and is a major interfering factor in the development of a durable seal between the tooth and composite restoration. Water leads to the formation of a variety of defects in dentin adhesives; these defects undermine the tooth-composite bond. Our group recently analyzed phase partitioning of dentin adhesives using high-performance liquid chromatography (HPLC). The concentration measurements provided by HPLC offered a more thorough representation of current adhesive performance and elucidated directions to be taken for further improvement. The sample preparation and instrument analysis using HPLC are, however, time-consuming and labor-intensive. The objective of this work was to develop a methodology for rapid, reliable, and accurate quantitative analysis of near-equilibrium phase partitioning in adhesives exposed to conditions simulating the wet oral environment. Analysis by Fourier transform infrared (FT-IR) spectroscopy in combination with multivariate statistical methods, including partial least squares (PLS) regression and principal component regression (PCR), were used for multivariate calibration to quantify the compositions in separated phases. Excellent predictions were achieved when either the hydrophobic-rich phase or the hydrophilicrich phase mixtures were analyzed. These results indicate that FT-IR spectroscopy has excellent potential as a rapid method of detection and quantification of dentin adhesives that experience phase separation under conditions that simulate the wet oral environment.

(C) 2013 Society for Applied Spectroscopy

*Author to whom correspondence should be sent. yeq@ku.edu.. 


\section{Keywords}

Attenuated total reflection-Fourier transform infrared; ATR FT-IR; Chemometrics; Dentin adhesive; Phase separation; Water; High-performance liquid chromatography; HPLC

\section{INTRODUCTION}

It has been reported that our current dentin adhesives are sensitive to excess moisture, leading to, for example, water blisters in adhesives placed on over-wet surfaces and phase separation with concomitant limited infiltration of the critical dimethacrylate component into the demineralized dentin matrix. ${ }^{1-5}$ Large fluid shifts that occur during the bonding process (solvent evaporation and light curing) may allow dentinal fluid to mix with the hydrophilic comonomers, creating nanoleakage pathways within the adhesives. ${ }^{6}$ To determine quantitatively the components in the hydrophobic-rich and hydrophilic-rich phases when exposed to over-wet environments, our group used high-performance liquid chromatography (HPLC) to analyze the well-separated phases of model dentin adhesive. ${ }^{7,8}$

The HPLC technique is time-consuming (typically taking 30-60 min) and labor-intensive. In addition, the increased requirements for sample handling during preparation can adversely affect the quality of the analysis. By contrast, Fourier transform infrared (FT-IR) spectroscopy is a very rapid (typically taking $30 \mathrm{~s}$ or less per sample) chemical fingerprinting technique that can potentially resolve the problems and produce results in under a minute after minimal sample preparation. Our aim in this study is therefore to investigate whether FT-IR spectroscopy is an accurate and valid technique for the detection and quantification of the phase compositions in both hydrophilic-rich and hydrophobic-rich phase samples.

When combined with appropriate multivariate statistical methods such as partial least squares (PLS) regression and principal component regression (PCR), FT-IR may be an ideal solution for the quantitative analysis of the phase compositions. Multivariate statistics is useful in spectral analysis because it facilitates the simultaneous inclusion of multiple spectra intensities, which greatly improves the precision and predictive ability of the analysis. ${ }^{9}$ To our knowledge, no work has been conducted to determine the compositions of phase-separated dentin adhesives using FT-IR spectroscopy with multivariate analysis.

\section{MATERIALS AND METHODS}

\section{Model Adhesive Composition and Sample Preparation}

The sample preparation has been described previously. ${ }^{7}$ In brief, the model adhesive consisted of hydroxyethylmethacrylate (HEMA; Acros Organics, NJ) and 2,2-bis[4-(2hydroxy-3-methacryloxypropoxy) phenyl]-propane (BisGMA; Polysciences Inc., Washington, PA) with a mass ratio of 45/55 (HEMA/BisGMA). ${ }^{4,10,11}$ Water (HPLC grade, W5SK-4; Fisher Scientific, Fair Lawn, NJ) was added into the neat resins with variable amounts, for example, 16, 33, and $50 \mathrm{wt} \%$. The concentration of water was based on the total final weight of the mixture. Previously we determined that $\sim 10 \%$ water is the threshold of water/resin (liquid/liquid) phase separation in HEMA/BisGMA formulations, with a mass 
ratio of 45/55. ${ }^{5}$ Shaking and sonication were required to yield well-dispersed solutions. These turbid mixtures were placed in $1.5-2.0 \mathrm{~mL}$ microcentrifuge tubes and further centrifuged (for $20 \mathrm{~min}$ at $10000 \mathrm{~g}$ ) to obtain clear separated solutions, a hydrophilic-rich aqueous phase and hydrophobic-rich resin phase according to the scheme (shown in Fig. 1). The lighter hydrophilic-rich phase (top layer) was transferred using a disposable micropipette. All the operations were carried out at ambient temperature $\left(24 \pm 1{ }^{\circ} \mathrm{C}\right)$.

\section{High-Performance Liquid Chromatograph}

The compositional analysis using reversed-phased high-performance liquid chromatography (RP-HPLC) has been described previously. ${ }^{7}$ In brief, the separated phase solutions were filtered using a Millipore centrifuge filter device (Ultrafree ${ }^{\circledR}-C L$, UFC4LCCOO 5000 NMWL; Millipore, Bedford, MA) and a centrifuge (Eppendorf MiniSpin Plus; Eppendorf, Hamburg, Germany) at $3000 \mathrm{rpm}$. A Shimadzu LC-2010 HTC HPLC system equipped with a photodiode array detector and EZStart chromatography software was used (Shimadzu, Columbia, MD). Separation was performed on a reverse-phase column, Phenomenex Luna 5 $\mu \mathrm{m} \mathrm{C18}, 4.6 \times 250 \mathrm{~mm}$ (Phenomenex, Torrance, CA) by elution with acetonitrile $\left(\mathrm{CH}_{3} \mathrm{CN}\right) / 20 \mathrm{mM}$ ammonium acetate buffer. The elution was started at a constant flow rate of $0.5 \mathrm{~mL} / \mathrm{min}$ with $\mathrm{CH}_{3} \mathrm{CN} / \mathrm{buffer}(35 / 65 \mathrm{v} / \mathrm{v}$ ) for $30 \mathrm{~min}$ and then ramped linearly to $100 \%$ $\mathrm{CH}_{3} \mathrm{CN}$ within $2 \mathrm{~min}$ and kept constant for $5 \mathrm{~min}$. Identification and quantitative analysis of the components were performed by comparison of the elution time and ultraviolet (UV) absorption peak intensity of the eluates with those of the individual, known standards. Typically the adhesive sample has to be diluted over thousand times to bring the analyte levels within the range of HPLC quantification. Triplicate injections of all the samples were carried out.

\section{Fourier Transform Infrared Instrumentation}

The separated phase solution was deposited without preparation onto a diamond crystal top plate of an attenuated total reflection (ATR) accessory (Pike, GladiATR, Pike Technologies, Madison, WI) covered with a mylar film. The infrared spectra were collected using a Spectrum 400 FT-IR spectrometer (Perkin Elmer, Waltham, MA). Sixteen scans were coadded over the range of 4000-650 $\mathrm{cm}^{-1}$ with a resolution of $4 \mathrm{~cm}^{-1}$. Air was taken as the reference for the background spectrum before each sample. After each spectrum, the ATR plate was cleaned in situ by scrubbing it with ethanol solution, which made drying the ATR possible. That no residue from the previous sample remained was verified by collecting a background spectrum and comparing it to the previous background spectrum. The samples were analyzed in triplicate, and the spectra were averaged for further analysis (three replicates from each sample were analyzed). All spectra were recorded at room temperature $\left(24 \pm 1^{\circ} \mathrm{C}\right)$ but without any nitrogen purge of the sample compartment.

\section{Chemometric Modeling}

Chemometrics is the use of mathematical and statistical methods to predict physical and chemical characteristics by indirect methods. Using this analysis, it is possible to predict the adhesive compositions once a calibration set of samples has been compiled. A total of 107 samples for the calibration models were prepared and tested using FT-IR. Prepared standard 
samples in different constituent-concentration ranges were selected for calibration model development using PLS regression and PCR. The software used for the principal component analysis was Spectrum QUANT+ expert Version 4.60 (Perkin Elmer). Calibration models were developed from spectra in absorbance units using PLS and PCR methods with the firstderivative transformed spectra. The optimum number of factors for calibration was selected on the basis of the predicted residual sum of squares, which should be minimized, along with the regression percentage variance. The developed calibration models were first crossvalidated in all cases to minimize the risk of overfitting and then used for testing or validation using the new data. The model prediction was tested by computing the standard error of prediction (SEP) and standard error of estimation (SEE); SEE can be described as the square root of the residual variance divided by the number of degrees of freedom, and SEP is the magnitude of the error expected when independent samples are predicted using the model.

\section{Data Analysis}

Data from the HPLC analysis were obtained in micrograms per milliliter for the minor components in the hydrophilic-rich phase. To convert from micrograms per milliliter to weight percent for the major components, the densities of the samples were measured using an analytical weighing balance with a resolution of $0.01 \mathrm{mg}$ (Mettler Toledo X205 dual range) and equipped with a density kit. ${ }^{12,13}$ The results from HPLC for the monomer and water content were compared against the results from FT-IR. For all experimental groups, the differences among compound concentrations were evaluated using one-way analysis of variance (ANOVA), together with Tukey's test at $a=0.05$ to identify significant differences.

\section{RESULTS}

Representative FT-IR spectra collected from HEMA, BisGMA, and water are presented in Fig. 2. The assignments of the spectral features for monomers are $1690-1740 \mathrm{~cm}^{-1}$ (carbonyl C=O stretching), 1635-1645 (aliphatic $\mathrm{C}=\mathrm{C}$ stretching), 1605-1609 (phenyl $\mathrm{C}=\mathrm{C}$ ), $1445-1457 \mathrm{~cm}^{-1}$ (ethyl group $\left[\mathrm{CH}_{2} \mathrm{CH}_{3}\right]$ ), $1402-1406 \mathrm{~cm}^{-1}$ (methylene group $\left[\mathrm{CH}_{2}\right]$ deformation), $1165-1180 \mathrm{~cm}^{-1}$ (C-O stretching), $830-836 \mathrm{~cm}^{-1}$ (C-C-O stretching), and 810 ( $\mathrm{C}=\mathrm{C}$ twist). Assignments for water $\left(\mathrm{H}_{2} \mathrm{O}\right)$ are $3000-3700 \mathrm{~cm}^{-1}$ (O-H stretching), $1500-1700 \mathrm{~cm}^{-1}$ (O-H deformation/scissors), and 650-900 $\mathrm{cm}^{-1}$ (water-hindered rotation). Overall, at the qualitative level, the spectra of HEMA and BisGMA look similar, and both overlap with characteristic peaks of water. The obvious visible differences between BisGMA and HEMA appear on the absorption bands related to the aromatic group.

When the absorption bands do not overlap, simple mathematical treatments, such as BeerLambert's calibration of peak heights or area with respect to the concentration of a single analyte, are possible. However, intense water bands in the infrared spectra obstruct the accuracy and sensitivity of quantitative analysis of multiple analytes. Because of the subtle differences previously highlighted, it was not possible to use simple visual inspection to quantify the levels of monomers and water. In this case, one of several multivariate methods 
such as PLS or PCR could be used in conjunction with the spectra to develop the multicomponent calibration models.

For the creation of a powerful model, a set of well-defined calibration samples is needed. Samples with a known composition that can be used for calibration can be easily prepared, in this case simply by mixing the components in a well-defined ratio. Figure 3 plots the water-HEMA-BisGMA ternary phase diagram, and all the standards shown in the singlephase region were used for calibration. There are two groups of standards, shown in Fig. 3, which were used for the hydrophobic-rich model and hydrophilic-rich model, separately.

Table I shows the summary statistics for PCR, PLS1, and PLS2 using the same training set associated with the hydrophobic-rich samples. In this case, the PLS1 and PLS2 algorithms outperformed PCR. In general, they resulted in a three-factor (or less) model with a high correlation coefficient. All samples were predicted with a similar level of accuracy, and the SEP values were about $0.002-0.004$ with percentage variance over $99 \%$. Figure 4 shows a representative calibration model, which was developed using a calibration set identical to that used for the PLS1 prediction models.

The PLS1 algorithm was also used for the hydrophilic-rich phase model. Table II shows the regression summary using the second group of standards and also one using both groups of standards. Note that the separation of the groups offers a six-factor (or less) model with less cross-validation error for the hydrophilic-rich model.

Both hydrophilic-rich and hydrophobic-rich phase compositions are calculated using HPLC and FT-IR chemometrics methods and are listed in Table III. It was found that the major components of the hydrophilic-rich phase were HEMA and water, and that the HEMA content ranged from 18.3 to $14.7 \mathrm{wt} \%$. The predicted values from the FT-IR chemometrics method matched very well with the HPLC results. It is noteworthy that the standard derivations of the calculated values from FT-IR chemometrics approach are usually lower than the ones from HPLC analysis.

\section{DISCUSSION}

The model adhesives were a simple mixture of one hydrophobic comonomer (BisGMA) and one hydrophilic comonomer (HEMA). The composition was based on conventional dentin adhesives. ${ }^{3,14}$ We investigated the ternary-phase diagram for the water-HEMA-BisGMA system ${ }^{7}$ and determined the phase-partition behavior of the experimental adhesives containing more than two comonomers with different hydrophobicity.

An FT-IR instrument, coupled with the powerful chemometrics-based analysis technique, was used to determine the composition of phase-separated dentin adhesives. Chemometric treatment of FT-IR spectra made it possible to obtain results similar to those obtained using the time-consuming and labor-intensive HPLC analytical technique. The results indicate that chemometric treatment of FT-IR spectra constitutes a fast and robust tool for the compositional analysis of phase-separated dentin adhesives. Infrared spectroscopy is a rapid technique; the spectral data are collected and interpreted within minutes. Usually, sample preparation is either not obligatory prior to spectral analysis or is minimal. It is possible to 
use chemometric statistical techniques in model development and classification. With the possibility of constructing a database containing a large number of known samples, further comparable tests should be very reliable.

Partial least squares is a commonly used multivariate regression method, especially in the field of spectroscopic study. The method is able to handle effectively the problem of multicollinearity, which is always present in spectroscopic data, whereas standard multivariate regression fails because of the rank deficiency problem. Partial least squares decomposes spectral data into loading and scores, building the corresponding calibration models from these new variables. ${ }^{15}$ This method requires that the analytes comply with Beer's law for the property to be measured. Partial least squares can predict either single predictors simultaneously using a PLS1 model or several predictors simultaneously using a PLS2 model. There is a substantial amount of literature devoted to the theoretical elucidation of the properties of the PLS algorithm. A good introduction to the method is given by Geladi and Kowalski. ${ }^{9}$

As expected, PLS leads to results that are slightly better than those from PCR because the PLS decomposition takes into consideration the variable to be predicted (monomer/water concentration) rather than simply decomposing the spectra into orthogonal components that are not necessarily related to the variable of interest. ${ }^{9}$ For instance, similar to what we observed using PCR for the hydrophobic-rich phase model, the best prediction error for BisGMA was obtained using a small model (three factors).

The sample concentrations of HEMA, BisGMA, and water predicted by PLS methods compare favorably with the data obtained using the HPLC method, presented in Table III. Therefore, the technique is highly plausible as an alternative to the standard procedure for the routine analysis of adhesive phase compositions. Compared to HPLC, the disadvantage of FT-IR chemometrics is the detection limit associated with spectroscopic analysis. The amount of BisGMA in the hydrophilic-rich phase sample is very small (converted to weight percent, the amount is less than 0.1 mass\%), which is lower than the detection limit of FT-IR chemometrics ( $\sim .2$ mass \%). However, the quantification of this minor component in the hydrophilic-rich samples is not critical for our research objective because this low concentration of BisGMA could not contribute significantly to the cross-linked density of the polymer network. ${ }^{16}$ These results support previous conclusions from our group that water-compatible cross-linkable comonomers must be included in adhesive formulations to address the defects that form in adhesives exposed to the wet oral environment. ${ }^{7,8,17-19}$

\section{CONCLUSION}

In this study, we demonstrated that in tertiary mixtures of dental monomer(s) and water, FTIR spectroscopy in combination with multivariate analysis, such as PLS, provides an accurate, simple, and rapid technique for the quantitative assessment of separated-phase composition. The sophisticated statistical approaches use the full or partial spectral region rather than unique and isolated analytical bands. We regard FT-IR spectroscopy, in combination with multivariate analysis, as a suitable alternative to HPLC for the detection and quantification of phase compositions in phase-separated dentin adhesives. 


\section{Acknowledgments}

This investigation was supported by Research Grants R01DE14392 and R01DE02202514392-08S1 and R01DE022054 from the National Institute of Dental and Craniofacial Research, National Institutes of Health, Bethesda, MD 20892.

\section{References}

1. Spencer P, Wang Y, Walker MP, Wieliczka DM, Swafford JR. Interfacial Chemistry of the Dentin/ Adhesive Bond. J. Dent. Res. 2000; 79(7):1458-1463. [PubMed: 11005728]

2. Tay FR, Pashley DH, Yoshiyama M. Two Modes of Nanoleakage Expression in Single-Step Adhesives. J. Dent. Res. 2002; 81(7):472-476. [PubMed: 12161459]

3. Spencer P, Wang Y. Adhesive Phase Separation at the Dentin Interface Under Wet Bonding Conditions. J. Biomed. Mater. Res. 2002; 62(3):447-456. [PubMed: 12209931]

4. Ye Q, Park JG, Topp E, Wang Y, Misra A, Spencer P. In Vitro Performance of NanoHeterogeneous Dentin Adhesive. J. Dent. Res. 2008; 87(9):829-833. [PubMed: 18719208]

5. Ye Q, Wang Y, Spencer P. Nanophase Separation of Polymers Exposed to Simulated Bonding Conditions. J. Biomed. Mater. Res. B. 2009; 88(2):339-348.

6. Hashimoto M, Ito S, Tay FR, Svizero NR, Sano H, Kaga M, Pashley DH. Fluid Movement Across the Resin-Dentin Interface During and After Bonding. J. Dent. Res. 2004; 83(11):843-848. [PubMed: 15505233]

7. Ye Q, Park J, Laurence JS, Parthasarathy R, Misra A, Spencer P. Ternary Phase Diagram of Model Dentin Adhesive Exposed to Over-Wet Environments. J. Dent. Res. 2011; 90(12):1434-1438. [PubMed: 21960682]

8. Ye Q, Park J, Parthasarathy R, Pamatmat F, Misra A, Laurence JS, Marangos O, Spencer P. Quantitative Analysis of Aqueous Phase Composition of Model Dentin Adhesives Experiencing Phase Separation. J. Biomed. Mater. Res. B. 2012; 100(4):1086-1092.

9. Geladi P, Kowalski BR. Partial Least-Squares Regression-a Tutorial. Anal. Chim. Acta. 1986; 185:1-17.

10. Ye Q, Park J, Topp E, Spencer P. Effect of Photoinitiators on the In Vitro Performance of a Dentin Adhesive Exposed to Simulated Oral Environment. Dent. Mater. 2009; 25(4):452-458. [PubMed: 19027937]

11. Ye Q, Spencer P, Wang Y, Misra A. Relationship of Solvent to the Photopolymerization Process, Properties, and Structure in Model Dentin Adhesives. J. Biomed. Mater. Res. A. 2007; 80(2):342350. [PubMed: 17001655]

12. Buruiana T, Buruiana EC, Melinte V, Colceriu A, Moldovan M. Urethane Dimethacrylate Oligomers for Dental Composite Matrix: Synthesis and Properties. Polym. Eng. Sci. 2009; 49(6): 1127-1135.

13. Feilzer AJ, Dauvillier BS. Effect of TEGDMA/BisGMA Ratio on Stress Development and Viscoelastic Properties of Experimental Two-Paste Composites. J. Dent. Res. 2003; 82(10):824828. [PubMed: 14514764]

14. Pashley EL, Zhang Y, Lockwood PE, Rueggeberg FA, Pashley DH. Effects of HEMA on Water Evaporation from Water-HEMA Mixtures. Dent. Mater. 1998; 14(1):6-10. [PubMed: 9972145]

15. Martens H. Factor Analysis of Chemical Mixtures: Non-Negative Factor Solutions for Spectra of Cereal Amino Acids. Anal. Chim. Acta-Com. 1979; 112(4):423-442.

16. Misra A, Parthasarathy R, Ye Q, Singh V, Spencer P. Swelling Equilibrium of Dentin Adhesive Polymers Formed on the Water-Adhesive Phase Boundary: Experiments and Micromechanical Model. Acta Biomater. 2013 in press. doi: 10.1016/j.actbio.2013.09.017.

17. Park J, Ye Q, Topp EM, Misra A, Kieweg SL, Spencer P. Effect of Photoinitiator System and Water Content on Dynamic Mechanical Properties of a Light-Cured bisGMA/HEMA Dental Resin. J. Biomed. Mater. Res. A. 2010; 93(4):1245-1251. [PubMed: 19827107]

18. Singh V, Misra A, Parthasarathy R, Ye Q, Park J, Spencer P. Mechanical Properties of Methacrylate-Based Model Dentin Adhesives: Effect of Loading Rate and Moisture Exposure. J. Biomed. Mater. Res. B. 2013 Paper in press. doi:10.1002/jbm.b.32963. 
19. Spencer P, Ye Q, Park J, Topp EM, Misra A, Marangos O, Wang Y, Bohaty BS, Singh V, Sene F, Eslick J, Camarda K, Katz JL. Adhesive/Dentin Interface: The Weak Link in the Composite Restoration. Ann. Biomed. Eng. 2010; 38(6):1989-2003. [PubMed: 20195761] 


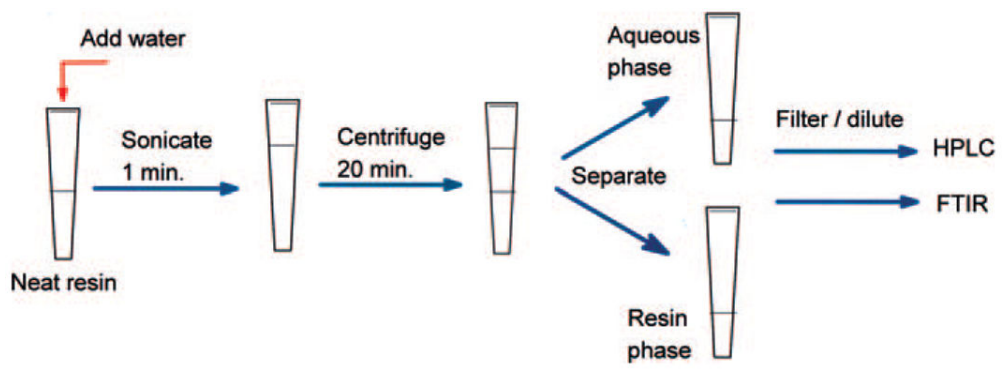

Fig. 1.

Scheme of sample preparation. 

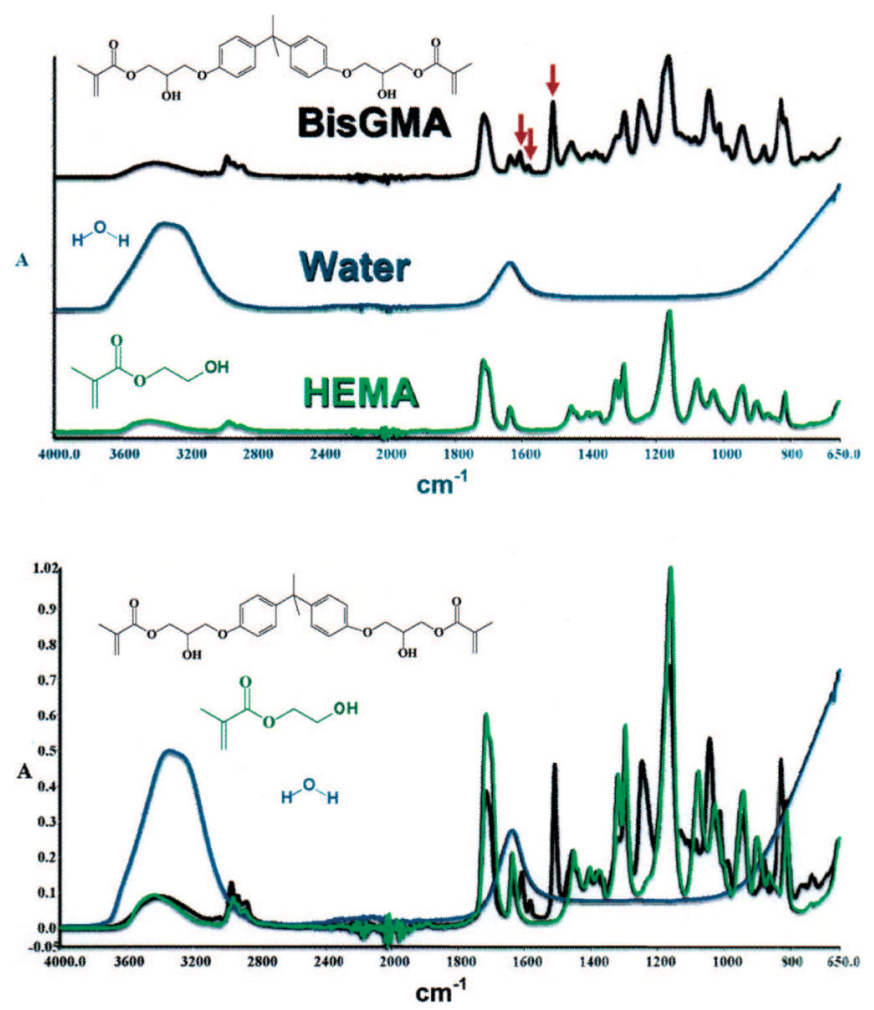

Fig. 2.

Attenuated total reflection FT-IR spectra of BisGMA (in black), HEMA (in green), and water (in blue). The obvious visible differences between BisGMA and HEMA appeared on the absorption bands related to the aromatic group (the arrows on the BisGMA spectrum). However, both the BisGMA and HEMA spectra overlap with characteristic peaks of water. 


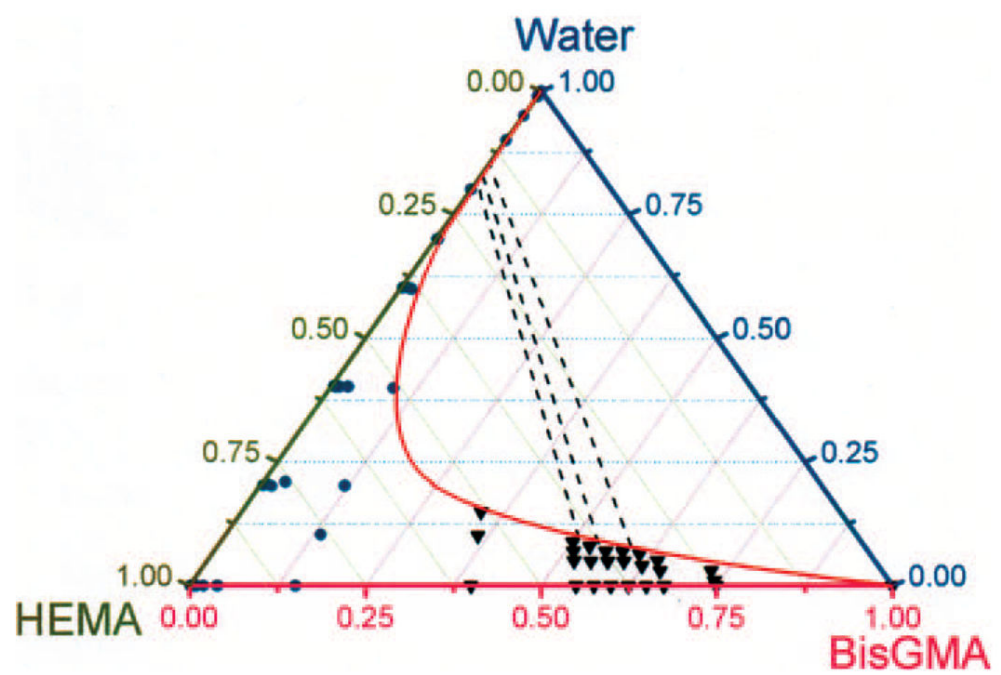

Fig. 3.

Standards in water-HEMA-BisGMA ternary-phase diagram used for the calibration model. The solid phase boundary line is drawn as a binodal curve, separating the single-phase and two-phase regions. Filled circles and triangles represent the standard samples used for the three-component models of hydrophobic-rich and hydrophilic-rich phases, respectively. Dashed lines represent three representative experimental tie lines. ${ }^{7,8}$ 


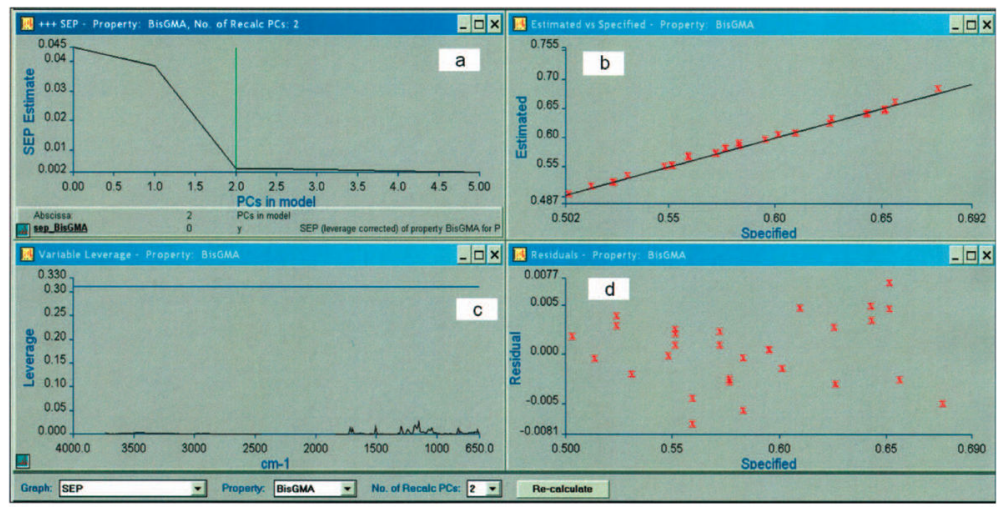

Fig. 4.

Representative calibration model. (a) Standard error of prediction graph, which gives a measure of the SEP associated with the model for a given number of principal components (PCs). The graph enables the selection of a different number of PCs to use to model the property (here, BisGMA) and a recalculation of the data. (b) Plot of estimated versus reference values. (c) Leverage graph displaying a cutoff line determined by the software, using parameters set by the operator. All standards that give results above the cutoff line are standard outliers. No significant outliers were found for any of the residual leverage plots. (d) Residual graph showing a random distribution of residuals about the 0 point of the $y$ axis. Any structure that is evident may indicate a problem with the model. 


\section{TABLE I}

Regression summary of different algorithms for three-component hydrophobic-rich phase model.

\begin{tabular}{lcccll}
\hline Property & Number of LVs & \\
& & Variance (\%) & SEE & SEP & Mean value \\
\hline PCR & & & & & \\
Water & 1 & 99.3 & 0.00253 & 0.00262 & 0.0362 \\
BisGMA & 5 & 99.8 & 0.00244 & 0.00253 & 0.586 \\
HEMA & 6 & 99.8 & 0.00225 & 0.00327 & 0.378 \\
PLS1 & & & & & \\
Water & 1 & 99.3 & 0.00252 & 0.00263 & 0.0362 \\
BisGMA & 2 & 99.4 & 0.00360 & 0.00378 & 0.586 \\
HEMA & 3 & 99.2 & 0.00400 & 0.00464 & 0.378 \\
PLS2 & & & & & \\
Water & 1 & 99.3 & 0.00253 & 0.00262 & 0.0362 \\
BisGMA & 2 & 99.4 & 0.00362 & 0.00379 & 0.586 \\
HEMA & 2 & 98.9 & 0.00461 & 0.00481 & 0.378 \\
\hline
\end{tabular}

${ }_{\text {LVs, latent variables. }}$ 


\section{TABLE II}

Regression summary of PLS1 algorithms using different ranges of standards.

\begin{tabular}{lccccl}
\hline Property & Number of $\mathbf{L V s}^{a}$ & Variance (\%) & SEE & SEP & Mean value \\
\hline Full range & & & & & \\
Water & 6 & 99.9 & 0.00341 & 0.00373 & 0.218 \\
BisGMA & 6 & 99.9 & 0.00388 & 0.00432 & 0.263 \\
HEMA & 7 & 99.9 & 0.00356 & 0.00397 & 0.505 \\
Hydrophilic phase & & & & & \\
Water & 5 & 99.9 & 0.00312 & 0.00350 & 0.295 \\
BisGMA & 6 & 99.9 & 0.00094 & 0.00138 & 0.031 \\
HEMA & 5 & 99.9 & 0.00336 & 0.00375 & 0.675 \\
Hydrophobic phase & & & & & \\
Water & 1 & 99.3 & 0.00252 & 0.00263 & 0.0362 \\
BisGMA & 2 & 99.4 & 0.00360 & 0.00378 & 0.586 \\
HEMA & 3 & 99.2 & 0.00400 & 0.00464 & 0.378 \\
\hline
\end{tabular}

$a_{\text {LVs, latent variables. }}$ 


\section{TABLE III}

Comparison of results obtained from HPLC and FT-IR chemometrics.

\begin{tabular}{|c|c|c|c|c|c|c|c|}
\hline \multirow[b]{2}{*}{ Initial water content $(\%)$} & \multirow[b]{2}{*}{ Method } & \multicolumn{3}{|c|}{$\underline{\text { Hydrophilic-rich-phase composition }}{ }^{a}$} & \multicolumn{3}{|c|}{$\underline{\text { Hydrophobic-rich-phase composition }}^{a}$} \\
\hline & & BisGMA $^{b}$ & HEMA & Water & BisGMA & HEMA & Water \\
\hline \multirow[t]{2}{*}{16.0} & HPLC & $0.054(0.002)$ & $18.3(0.8)$ & $81.6(0.6)$ & $50.8(0.5)$ & $39.7(0.4)$ & $9.5(0.3)$ \\
\hline & FT-IR & N/A & $18.0(0.1)$ & $82.0(0.1)$ & $50.9(0.1)$ & $39.9(0.2)$ & $9.4(0.1)$ \\
\hline \multirow[t]{2}{*}{33.0} & HPLC & $0.030(0.002)$ & $16.8(0.7)$ & $83.1(0.8)$ & $53.9(0.3)$ & $37.4(0.3)$ & $8.6(0.2)$ \\
\hline & FT-IR & N/A & $16.5(0.1)$ & $83.5(0.1)$ & $53.9(0.2)$ & $37.5(0.1)$ & $8.5(0.1)$ \\
\hline \multirow[t]{2}{*}{50.0} & HPLC & $0.014(0.001)$ & $14.7(1.2)$ & $85.3(1.1)$ & $58.9(0.4)$ & $33.1(0.3)$ & $7.9(0.2)$ \\
\hline & FT-IR & N/A & $14.5(0.2)$ & $85.5(0.2)$ & $58.8(0.2)$ & $33.2(0.1)$ & $7.9(0.1)$ \\
\hline
\end{tabular}

The amount of BisGMA in aqueous phase is very small $(<0.06 \%)$ and is lower than the detection limit of FT-IR chemometrics (about $0.2 \%)$.

$a_{n=3}$. Values in parentheses are weight percent.

${ }^{b}$ N/A, not available. 